\title{
Momentum transport and flow damping in the reversed-field pinch plasma
}

\author{
A. F. Almagri, J. T. Chapman, C. S. Chiang, D. Craig, D. J. Den Hartog, C. C. Hegna, \\ and S. C. Prager \\ Department of Physics, University of Wisconsin, Madison, Wisconsin 53706
}

(Received 24 February 1998; accepted 5 August 1998)

\begin{abstract}
A biased electrode is used in the Madison Symmetric Torus (MST) reversed-field pinch [Fusion Technol. 19, 131 (1991)] to manipulate plasma flow in order to study flow damping and momentum transport. Finite radial conductivity allows a radial current, which provides the toroidal torque that spins up the plasma. The applied torque is balanced by a viscous force that opposes toroidal flow acceleration. From the plasma flow damping the viscosity is inferred to be anomalous. The radial transport of toroidal momentum is comparable to that of particles and energy, and is consistent with transport by stochastic magnetic field lines. (C) 1998 American Institute of Physics.
\end{abstract}

[S1070-664X(98)02011-4]

\section{INTRODUCTION}

Magnetic and electrostatic fluctuations play an important role in the radial transport of energy and particles in the Madison Symmetric Torus (MST) reversed-field pinch (RFP) plasma where the transport was measured to be anomalous. Results ${ }^{1-3}$ have shown that, in the edge of MST, electrostatic fluctuations are important and control particle transport; however, neither the electrostatic nor the magnetic fluctuations can account for the large energy flux. In the core of MST, magnetic fluctuations were identified ${ }^{3,4}$ as the driving source of the large particle and heat transport. The particle diffusion coefficient $D\left(a^{2} / 4 \tau_{p}\right)$ and the thermal diffusion coefficient $\chi\left(a^{2} / 4 \tau_{E}\right)$ are large $\left(10-50 \mathrm{~m}^{2} / \mathrm{s}\right)$. These coefficients are larger than classical diffusion coefficients $\left(\rho_{e}^{2} \nu_{e} \leqslant 3 \times 10^{-2} \mathrm{~m}^{2} / \mathrm{s}\right.$ and $\left.\rho_{1}^{2} \nu_{i} \leqslant 0.6 \mathrm{~m}^{2} / \mathrm{s}\right)$. However, these measured values are smaller than that given by the quasilinear stochastic diffusion rate ${ }^{5}$ evaluated at the electron thermal speed.

Magnetic fluctuations in MST are dominated by lowfrequency components $(10-30 \mathrm{kHz})$, which contain about $90 \%$ of the power. These fluctuations identified ${ }^{6}$ as being due to the tearing instability, are composed of several spatial modes with $m=1, n=5-10$. The measured frequency in the experiment is interpreted as the Doppler shift frequency $(\omega=k \cdot v)$ due to plasma rotation in the lab frame. The rms amplitude of the magnetic fluctuation is generally $1 \%-2 \%$ of the mean field. The parallel correlation length of the dominant magnetic fluctuation in the low-frequency range $(10<f<30 \mathrm{kHz})$ is measured ${ }^{7}$ to be about $3.5-4 \mathrm{~m}$ and at the high-frequency range $(>30 \mathrm{kHz})$ the correlation length is about $1 \mathrm{~m}$.

In this paper, we describe the results of experiments, designed to infer the radial transport of toroidal momentum, related to the plasma viscosity. We measure the response of the plasma flow to impulsive changes in the flow, which are imposed with a biased electrode. The plasma flow is inferred by two techniques. First, we measure the impurity ion flow with Doppler spectroscopy, which provides limited information on the radial profile of the flow. Second, we measure the toroidal phase velocity of the resonant tearing modes with a magnetic coil array, and identify the phase speed of each Fourier component with the plasma flow speed at the corresponding resonant surface.

To avoid an unnecessary complication associated with locked discharges we have chosen to impulsively spin up an already rotating discharge, which is the standard case in MST. The parameters of the discharges studied in this paper are chosen to avoid locked discharges and to reduce the plasma damage to the biased electrode. These plasmas have the standard flow profile, where the plasma is flowing at about $10-20 \mathrm{~km} / \mathrm{s}$ at the core with a decreasing flow out to the edge that can be near zero or at a negative flow $(-5 \mathrm{~km} / \mathrm{s})$. This small edge flow is consistent with measured radial electric field. ${ }^{8}$ The reversal in the flow may be near the toroidal field reversal radius but does not appear to be coupled to it. However, MST has another class of discharges where the plasma flow is zero in the lab frame. These discharges become locked due to the interaction with the selfgenerated field errors at the poloidal gap. ${ }^{9}$ These locked discharges are more likely to be produced at high levels of plasma current (400-600 kA) than the levels of plasma current studied in this paper $(\sim 200 \mathrm{kA})$.

When the bias is removed, the plasma evolves back to the flow state, which existed previously. Hence, the total plasma momentum decreases. This can occur for either of two reasons. First, the wall or edge plasma may impose a boundary condition that the edge flow be zero (for example, from friction between the rotating plasma and refluxing particles). The core plasma will then relax on the viscous time scale, which we measure. This momentum loss mechanism can occur in a toroidally symmetric system. The plasma momentum is transferred elsewhere, such as to the wall. Second, asymmetry in the external magnetic configuration caused by either field errors or wall currents induced by magnetic fluctuations in the plasma can cause an external torque to reduce the plasma momentum. In either case, the relaxation of the velocity profile reveals the internal plasma viscosity.

We find that the plasma response time to either a sudden turn-on or a sudden turn-off of the edge flow is anomalous. 
Thus, the radial transport of momentum, and the plasma viscosity, exceeds the classical value by about two orders of magnitude. The momentum transport coefficient is about equal to that for energy and particle, which may be expected, since all three are driven by the same mechanism of magnetic fluctuations. However, the momentum transport coefficient is consistent with the heuristic values predicted for transport from the stochastic magnetic field.

\section{PROBE DESCRIPTION, DIAGNOSTICS, AND EXPERIMENTAL SETUP}

Fast changes in plasma flow are induced by a biased electrode inserted into the edge plasma. The plasma-exposed part of the active element (molybdenum) is in the form of a cylinder that is $3 / 4 \mathrm{in}$. in diameter and $1 / 8 \mathrm{in}$. thick. The rest of the probe is isolated from the plasma with a boron nitride shroud that is $12 \mathrm{in.} \mathrm{long}$ and $1 \mathrm{in}$. in diameter, which allows the insertion of the active element deep into the plasma. The probe can be biased and unbiased at preset times in the discharge with a fast turn-on and a fast turn-off. This turnon and turn-off of biasing is done within a very short time (less than $100 \mu \mathrm{s})$. The probe is typically inserted to a radius $r=37 \mathrm{~cm}(r / a=0.71)$ and biased relative to the conducting shell with a square pulse $10 \mathrm{~ms}$ long. We signify the plasma that is inside the probe tip $(r / a \leqslant 0.71)$ as the core plasma and the plasma that is outside the probe tip $(r / a \geqslant 0.71)$ as the edge plasma. The probe can be biased in both polarities up to $\pm 400 \mathrm{~V}$, which is a limit set by the power supply.

The presence of the electrode with or without bias does not affect the magnetic configuration, as measured by the reversal parameter $F \equiv B_{t}(r=a) /\left\langle B_{t}\right\rangle$ and pinch parameter $\theta \equiv B_{\theta}(r=a) /\left\langle B_{t}\right\rangle$. The dynamo activity is also not affected, as indicated by the presence of the sawteeth activity. Biasing of the electrode does not effect the amplitude and spectrum of the magnetic fluctuations. The magnetic fluctuations and the dynamo mechanism are very robust features of the RFP. However, with a negatively biased electrode we do observe reduction in the electrostatic fluctuations and the associated improvements in the plasma particle confinement, ${ }^{8}$ which is very similar to tokamak results. Also similar to tokamak results, the positive bias of the electrode does not produce a strong enhancement in the particle confinement. With this impulsive biasing technique, we can successfully manipulate the plasma flow. However, to prevent and reduce the degradation of plasma, which can be caused by insertion of a material object into the plasma volume, the probe must be cleaned and thoroughly conditioned before it can be biased.

The plasma flow profile is inferred by two techniques. First, a crude flow profile has been obtained by measuring the toroidal flow of two impurity species (CV and CIII) with a Doppler duospectrometer. ${ }^{10}$ The Doppler broadened and shifted emission from the $\mathrm{Cv}$ impurity ions in these plasmas is dominated by ions at $r / a \approx 0.4$ (core plasma) while the emission from CIII is dominated by ions at $r / a \approx 0.85$ (edge plasma). Second, the phase speed of the resonant tearing modes is measured with an array of magnetic pickup coils. A toroidal array of 32 magnetic pickup coils that are equally spaced in the toroidal direction measures the magnetic field

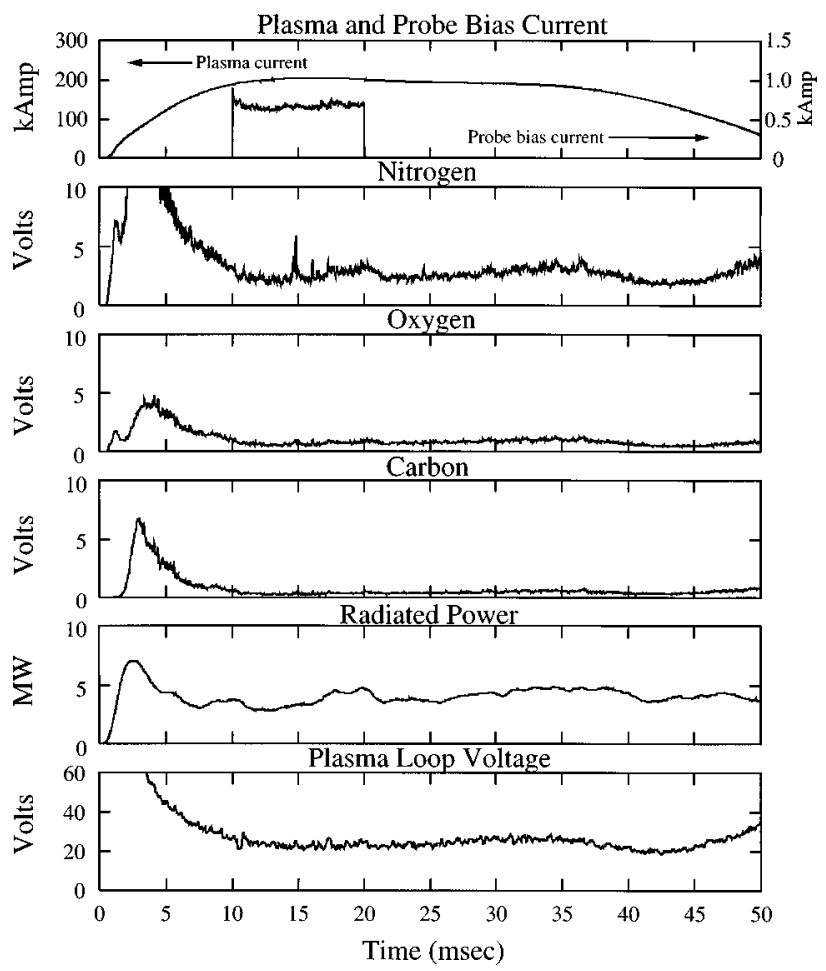

FIG. 1. Plasma current, bias current, and impurity ion signals of nitrogen, oxygen, and carbon lines, and the radiated power and plasma loop voltage, all show that no deleterious effects of the biasing on the plasma.

at the edge. The magnetic fluctuation produced by the core resonant tearing modes is measured as a function of time and toroidal angle. The fluctuation is Fourier decomposed into its toroidal components, which are resonant at different radial locations. The phase of each Fourier component as a function of time then provides a radial profile of the mode phase velocity from $\omega=k \cdot \nu$. The radial profile of the phase velocity of these modes reflects the radial profiles of the plasma flow.

The MST is a large reversed-field pinch with a close fitting, $0.05 \mathrm{~m}$ thick, conducting shell. The major radius $R$ $=1.5 \mathrm{~m}$ and the minor radius $a=0.52 \mathrm{~m}$. For the experiments discussed here, MST was operated in a low-current hydrogen plasma with plasma current $I_{p} \approx 200 \mathrm{kA}$, electron density $n_{e} \approx 8 \times 10^{18} \mathrm{~m}^{-3}$, electron temperature $T_{e}$ $\approx 150 \mathrm{eV}$, ion temperature $T_{i} \approx 120 \mathrm{eV}$, reversal parameter $F \approx-0.15$, and pinch parameter $\theta \approx 1.8$

\section{EFFECTS ON PLASMA PARAMETERS}

With a well-conditioned probe, there is no degradation of plasma parameters when the probe is biased. Figure 1 shows the plasma current and the bias current, which is extracted at the flat top portion of plasma, current. Figure 1 also shows the time histories of several edge impurity signals such as nitrogen, oxygen, and carbon averaged over about 20 discharges. These signals show no increase in amplitude when the probe is biased and maintained for $10 \mathrm{~ms}$ duration. Also shown in Fig. 1 are the resistive loop voltage and the total radiated power of the plasma averaged over about 20 


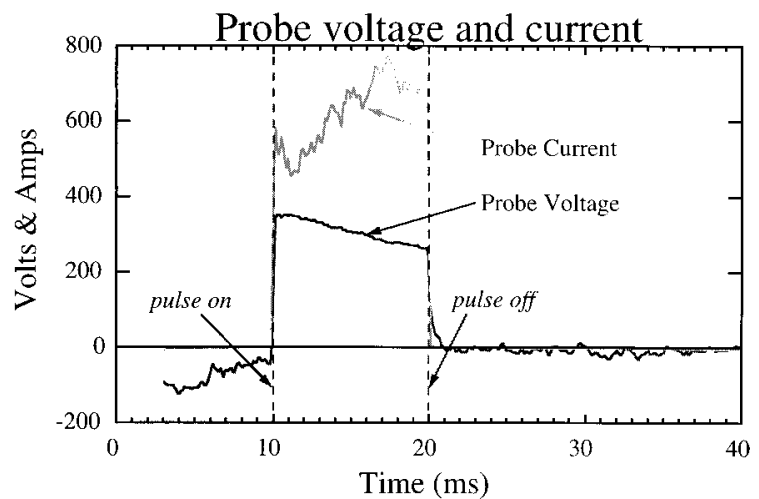

FIG. 2. The bias voltage and the injected current of the bias probe during fast turn-on and fast turn-off.

discharges. These parameters show that the biasing of the probe has no significant effect on the plasma radiation or the plasma resistivity.

\section{PLASMA FLOW AND VISCOUS DAMPING}

During impulsive biasing, the flow of both species of carbon and the phase velocity of the resonant tearing modes respond quickly. As will be shown, the spin-up of the toroidal flow is a measure of the radial transport of toroidal momentum and the slowing-down time of the flow is proportional to the viscous damping.

Figure 2 shows the bias voltage and the extracted current of the probe. After the first $2 \mathrm{~ms}$ of bias, the extracted current starts to increase although the bias voltage is decreasing indicating an enhanced radial conductivity of the plasma with bias. Figure 3 shows the time history of the ion $(\mathrm{Cv})$ toroidal flow in the core with and without plasma biasing. The biasing pulse starts $10 \mathrm{~ms}$ into the discharge and lasts for $10 \mathrm{~ms}$, which is chosen to be much longer than the particle or energy confinement time scales $(\sim 1 \mathrm{~ms})$. With bias, the plasma

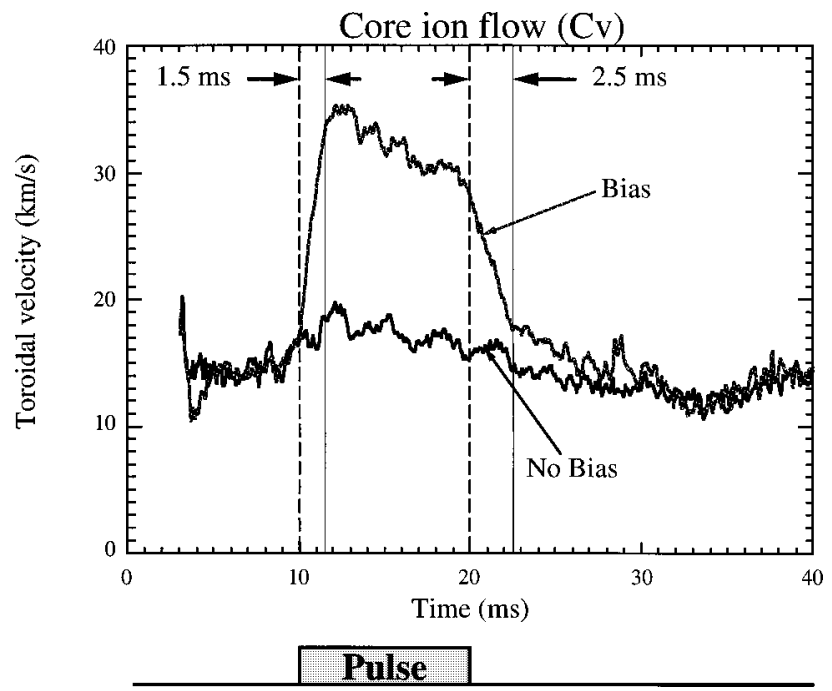

FIG. 3. The toroidal flow, of $\mathrm{Cv}$ impurity, with and without bias, shows that the core plasma accelerates within $1.5 \mathrm{~ms}$ to about double the toroidal flow when the probe is not biased. When the bias is turned off at $20 \mathrm{~ms}$ the plasma slows down in about $2.5 \mathrm{~ms}$ due to viscous damping.

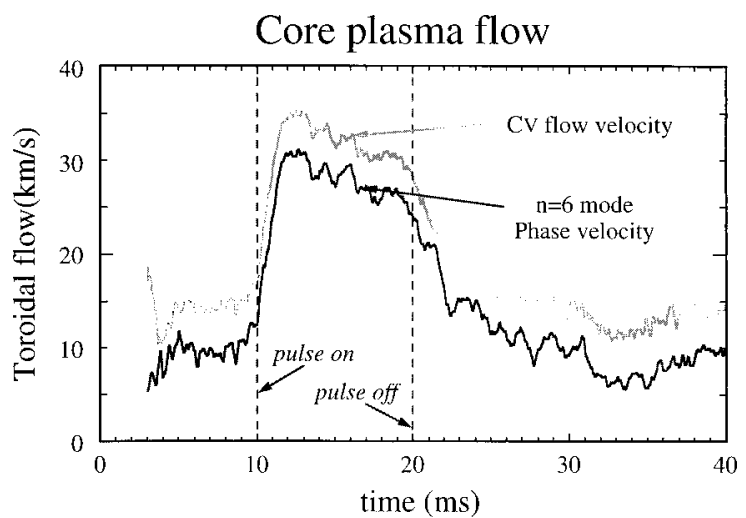

FIG. 4. The toroidal flow with bias as measured by both the $\mathrm{CV}$ impurity flow and the phase velocity of the core resonant $n=6$ mode.

flow increases from about 15 to about $35 \mathrm{~km} / \mathrm{s}$ in about 1.5 ms duration. The toroidal flow is maintained at this level for the duration of the biasing pulse. At $20 \mathrm{~ms}$ the bias pulse is turned off very fast and the driving term removed, the toroidal flow starts to decay back to the no-bias level in about 2.5 ms due to viscous damping.

The core plasma that extends out to $r=0.37 \mathrm{~m}$ slows down in about a $2.5 \mathrm{~ms}$ period. The kinematic viscosity, inferred from the plasma slowing-down time, defined by $\nu_{\perp} \sim(\Delta r)^{2} / \tau_{\text {sd }}$, where $\tau_{\text {sd }}$ is the measured slowing-down time and $\Delta r$ is the radial extent of the core region, indicates that the core average viscosity is about $55 \mathrm{~m}^{2} / \mathrm{s}$, which is anomalous. This scaling of the viscosity comes from a simplified momentum equation for the toroidal flow, given by

$$
\rho \partial v_{\phi} / \partial t=\rho \nu_{\perp} \nabla^{2} v_{\phi},
$$

where $v_{\phi}$ is the plasma toroidal flow, $\rho$ is the mass density, and $\nu_{\perp}$ is kinematic viscosity.

The expected classical kinematic viscosity for typical discharge parameters is small by two orders of magnitude $\left(\nu_{\perp} \sim \rho_{i}^{2} / \tau_{i} \sim 0.6 \mathrm{~m}^{2} / \mathrm{s}\right)$, and the classical slowing-down time scale $\left(\tau_{\mathrm{sd}} \sim(\Delta r)^{2} / \rho_{i}^{2} \nu_{i} \approx 250 \mathrm{~ms}\right)$ is very long compared to the measured slowing time scale. The slowing-down time

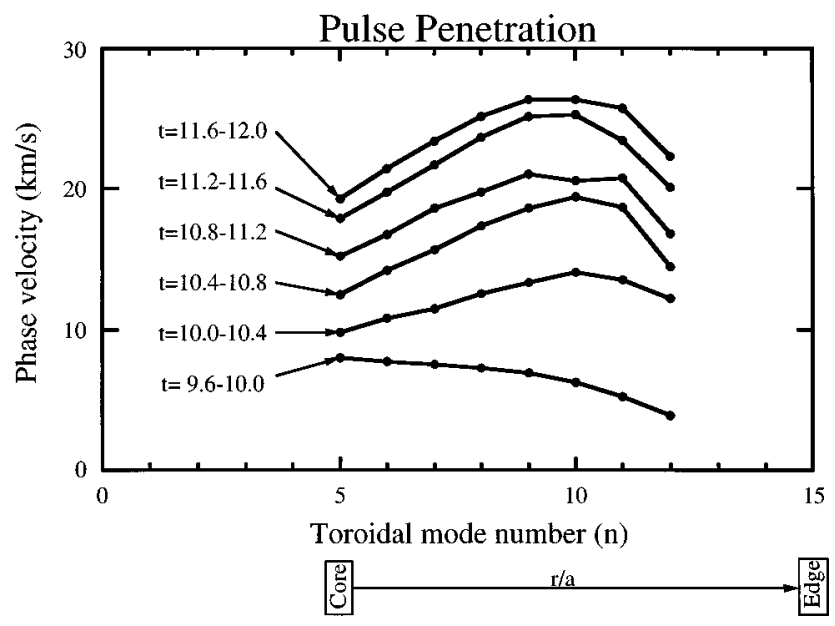

FIG. 5. The rotating frequency of the tearing modes $(n=5-12)$ changes as the plasma is biased shown the penetration of the toroidal flow from the edge (location of the biased probe) to the core. 


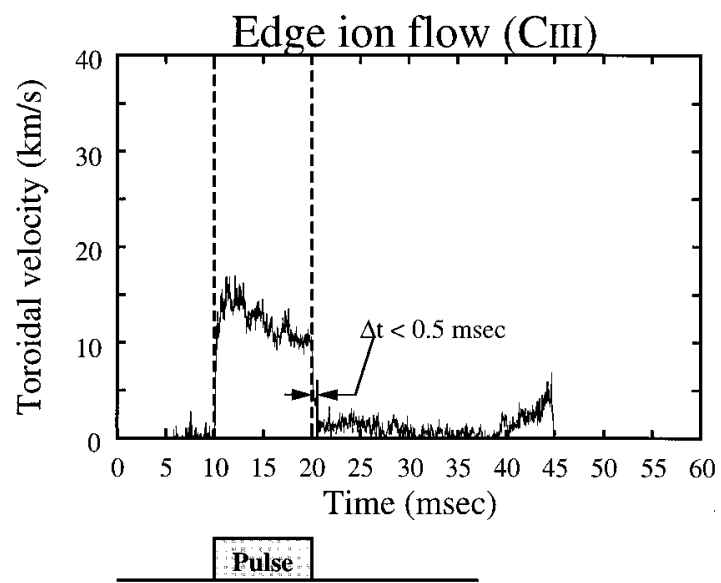

FIG. 6. The toroidal flow, of CIII with bias in the edge plasma. The plasma edge accelerate within $100 \mu$ s from -5 to about $+15 \mathrm{~km} / \mathrm{s}$. When the bias is turned off at $20 \mathrm{~ms}$, the plasma slows down in less than $0.5 \mathrm{~ms}$ due to viscous damping.

scale of the core flow is independent of plasma density, which is inconsistent with the classical processes.

The phase velocity of the resonant tearing modes, $(m=1, n=5-12)$, changes with biasing, reflecting the change in the local plasma flow. Figure 4 compares the toroidal flow velocity as measured by $\mathrm{CV}$ impurity and by the phase velocity of the core resonant $n=6$ mode. This figure shows how well the local phase velocity tracks the impurity ion flow. Figure 5 shows the radial profile of the toroidal phase velocity of the resonant tearing modes at six time windows that are $0.4 \mathrm{~ms}$ wide. The first time window is from 9.6 until $10 \mathrm{~ms}$ and shows the flow profile before biasing. The other five time windows show the change in the profile during the first $2 \mathrm{~ms}$ of the biasing pulse. The modes $(n=10,11,12$ near $r / a \sim 0.7)$ that are resonant nearest the probe are accelerated first, followed by an acceleration of the inner modes $(n=5,6,7$ near $r / a \sim 0.3)$. As time progresses, the inner modes catch up to outer modes. Figure 4 clearly shows that toroidal flow diffuses radially inward from the edge, where the probe is located, in about a $1.5 \mathrm{~ms}$ time period. This is similar to the time duration needed for the core plasma flow to reach its peak, as shown in Fig. 3, which is representative of the plasma flow at $r / a \sim 0.4$. This time scale of the radial transport of the toroidal flow is similar to the energy and particle transport time scale.

Figure 6 shows the time history of the edge flow, the outer $15 \mathrm{~cm}$ of the plasma, using the CIII impurity line. Without bias, the edge flow is typically negative and small in magnitude (about $-5 \mathrm{~km} / \mathrm{s}$ ). With bias, edge flow slows down and becomes positive in direction (about $15 \mathrm{~km} / \mathrm{s}$ ) on a faster time scale; then the particle confinement time in a standard discharge $\left(\tau_{p} \sim 1.5 \mathrm{~ms}\right)$. The slowing down of the edge region, in less than $0.5 \mathrm{~ms}$, results in an edge kinematic viscosity of about $45 \mathrm{~m}^{2} / \mathrm{s}$, which is also anomalous. The observed change in the edge flow with bias is about $20 \mathrm{~km} / \mathrm{s}$, which is consistent with the $E \times B$ flow $\left(E_{r} / B \sim 25 \mathrm{~km} / \mathrm{s}\right)$. This flow perturbation of $20 \mathrm{~km} / \mathrm{s}$ is then radially transported to the core where the flow increases by about $20 \mathrm{~km} / \mathrm{s}$ in about $1.5 \mathrm{~ms}$.

The anomalous viscosity in the core of MST is consistent with the estimates of magnetically driven transport of momentum, ${ }^{11}$ as given by

$$
\nu_{\perp} \sim(\Delta r)^{2} / \tau_{\mathrm{sd}}=\left(\frac{\delta B_{r}}{B}\right)^{2} L C_{s} \approx 50 \mathrm{~m}^{2} / \mathrm{s},
$$

where $\delta B_{r}$ is the perturbed magnetic field due to tearing modes, $L$ is the parallel correlation length, and $C_{s}$ is the plasma sound speed. In the edge of MST, the viscosity is also anomalous, but the source of momentum transport is not known.

\section{SUMMARY AND CONCLUSIONS}

In summary, plasma flow is manipulated and modified to investigate flow damping and viscosity in the RFP. Radial currents from a biased electrode provide the toroidal torque that spins up the plasma against the viscous forces opposing the toroidal flow. The plasma flow damps much faster (in about $2 \mathrm{~ms}$ ) than the classical estimates (in about 250 $\mathrm{ms}$ ), and this time is independent of plasma density. We conclude that in the core of MST, particle, heat, and momentum transport are all anomalous and are likely driven by the magnetic fluctuations. The anomalous core viscosity is consistent with the estimates due to stochastic magnetic field lines. In the edge of MST, only particle transport is understood. The sources of heat and momentum transport are not known but they may be electrostatic in character.

\section{ACKNOWLEDGMENTS}

The authors acknowledge and are grateful for many discussions and suggestions from MST members. This work has been supported by the U.S. Department of Energy.

${ }^{1}$ T. D. Rempel, A. F. Almagri, S. Assadi, D. J. Den Hartog, S. A. Hokin, S. C. Prager, J. S. Sarff, W. Shen, K. L. Sidikman, C. W. Spragins, J. C. Sprott, M. R. Stoneking, and E. J. Zita, Phys. Fluids B 4, 2136 (1992).

${ }^{2}$ T. D. Rempel, C. W. Spragins, S. C. Prager, S. Assadi, D. J. Den Hartog, and S. A. Hokin, Phys. Rev. Lett. 67, 1438 (1991).

${ }^{3}$ M. R. Stoneking, S. A. Hokin, S. C. Prager, G. Fiksel, H. Ji, and D. J. Den Hartog, Phys. Rev. Lett. 73, 549 (1994).

${ }^{4}$ G. Fiksel, S. C. Prager, W. Shen, and M. R. Stoneking, Phys. Rev. Lett. 72, 1028 (1994).

${ }^{5}$ A. B. Rechester and M. N. Rosenbluth, Phys. Rev. Lett. 40, 38 (1978).

${ }^{6}$ S. Assadi, S. C. Prager, and K. L. Sidikman, Phys. Rev. Lett. 69, 281 (1992).

${ }^{7}$ M. R. Stoneking, Ph.D. thesis, University of Wisconsin-Madison, 1994.

${ }^{8}$ D. Craig, A. F. Almagri, J. K. Anderson, J. T. Chapman, C.-S. Chiang, N. A. Crocker, D. J. Den Hartog, G. Fiksel, S. C. Prager, J. S. Sarff, and M. R. Stoneking, Phys. Rev. Lett. 10, 1865 (1997).

${ }^{9}$ A. F. Almagri, S. Assadi, S. C. Prager, J. S. Sarff, and D. W. Kerst, Phys. Fluids B 4, 4080 (1992).

${ }^{10}$ D. J. Den Hartog and R. J. Fonck, Rev. Sci. Instrum. 65, 3238 (1994).

${ }^{11}$ J. M. Finn, P. N. Guzdar, and A. A. Chernikov, Phys. Fluids B 4, 1152 (1992). 